\title{
Applications and development of permeability imaging in ischemic stroke (Review)
}

\author{
HUI CHEN ${ }^{1}$, GUANGMING ZHU $^{1}$, NAN LIU ${ }^{1}$, YING LI $^{1}$ and YONGHONG XIA ${ }^{2}$ \\ ${ }^{1}$ Department of Neurology, Military General Hospital of Beijing PLA, Beijing 100700; ${ }^{2}$ Department of \\ Critical Care Medicine, Yantai Yuhuangding Hospital, Yantai, Shandong 264000, P.R. China
}

Received November 7, 2016; Accepted January 6, 2017

DOI: $10.3892 /$ etm.2018.6454

\begin{abstract}
Brain permeability imaging techniques are specific for the assessment of blood-brain barrier integrity. The present review article primarily focuses on the application of permeability imaging in cases of ischemic stroke. The permeability maps may be used to predict future hemorrhagic transformation in patients following acute ischemic stroke, that have been treated with tissue plasminogen activator (tPA) or recanalization therapy. The permeability imaging would help make the clinical decision to administer tPA following acute ischemic stroke or not, which is not only due to the current 3-4.5 h time window. Additionally, permeability imaging may also be used to evaluate the collateral circulation in the perfusion and permeability of the ischemic area of the brain.
\end{abstract}

\section{Contents}

1. Introduction

2. Prediction of HT following acute ischemic stroke

3. Assessment of intracranial collateral circulation

4. Conclusions

\section{Introduction}

Multimodal imaging techniques, including various imaging sequence parameters, such as vascular, diffusion, perfusion imaging and spectral analysis are now routinely utilized in clinical practice. The living imaging technologies capable of quantitatively measuring the blood-brain-barrier permeability (BBBP) in vivo are collectively known as the brain permeability imaging techniques and are specific for the assessment

Correspondence to: Dr Yonghong Xia, Department of Critical Care Medicine, Yantai Yuhuangding Hospital, 20 East Yuhuangding Road, Yantai, Shandong 264000, P.R. China

E-mail: siece450@sina.com

Key words: hemorrhagic transformation, acute ischemic stroke, blood brain barrier permeability, intracranial collateral circulation of the integrity of the blood-brain barrier (BBB) $(1,2)$. The use of permeability imaging for making therapeutic decisions and prognostication in neurological disorders has become a major focus of research worldwide (2). In the present review article, the application of permeability imaging in cases of ischemic stroke is assessed.

In the acute and sub-acute phases of stroke, the BBB exhibits differing degrees of defects, which participate in the pathophysiological processes of tissue damage and repair; for example, an increased brain edema and initiation of cerebral hemorrhagic transformation (HT). A previous study indicated that the earliest evidence of brain edema may be identified $30 \mathrm{~min}$ after cerebral ischemia (3). The BBB, however, remains intact for up to $2 \mathrm{~h}$ following ischemia, indicating that the earliest cerebral edema may be primarily associated with cell edema (3). Studies in rats have revealed that, following continuous ischemia, the increase in the BBBP to small molecules occurs from the third day, which is also the time when brain edema peaks $(4,5)$. This suggests that the brain edema at this stage is primarily associated with increased permeability of the BBB to small molecules. Angiogenesis is the manifestation of vascular remodeling during stroke recovery. BBB leakage during the early stage of angiogenesis requires further investigation, as it is a new research field (6). $\mathrm{BBB}$ assessment in angiogenesis may help to predict the possibility of HT and judge the prognosis. In other diseases, such as tumors, BBB analysis may help to distinguish the grade of malignancy. As the newborn vessels constitute the secondary and tertiary collateral circulation pathways in the brain, the assessment of angiogenesis may reflect the condition of the secondary and tertiary collateral circulations within the brain. That the collateral circulation is closely associated with stroke prognosis, evaluation of the collateral circulation is currently a major focus of research (7). Thus, the present review summarizes the application of permeability imaging in the prediction of HT and evaluation of the collateral circulations following acute ischemic stroke.

\section{Prediction of HT following acute ischemic stroke}

A number of large international stroke studies reported a HT rate of 2.4-8.8\% following the use of tissue plasminogen activator (tPA) $(8,9)$. Other studies reported the occurrence of HT in $8-9.9 \%$ of patients who have undergone mechanical 
angioplasty combined with intravenous or intra-arterial thrombolysis $(10,11)$. All reperfusion therapies that have proven effective for improving the functional outcomes of patients with ischemic stroke are clearly associated with an increased risk of HT, which may be fatal $(12,13)$. Therefore, prior to making diagnostic and treatment decisions for patients with ischemic stroke, the risk of HT should be fully assessed in order to avoid bleeding as much as possible and, particularly, avoid the onset of symptomatic intracerebral hemorrhage, which is critical for a favorable prognosis.

Previously, the occurrence of HT was primarily predicted according to the severity and type of stroke, the size and location of the infarcts, the reperfusion time, the time and method of thrombolytic therapy and the history of previous anticoagulant therapy (4). However, these evaluation methods may only indicate certain outcomes and cannot predict the site of hemorrhage. Various studies have attempted to directly assess the risk of HT and its site according to the size or extent of ischemia exhibited in perfusion imaging. Souza et al (14) hypothesized that the brain regions with a relative cerebral blood flow of $<0.48$ and a relative mean transit time $>11.3$ have a higher risk of HT. The predictive ability of this method may be more effective than the magnetic resonance (MR) diffusion-weighted imaging (DWI) (15). In essence, however, this prediction method is no different from the evaluation based on the National Institutes of Health Stoke Scale score, infarct volume or collateral circulation (16). In addition, it has no prominent localization significance, is not highly specific or quantitative and cannot determine the association among the perfusion levels, the mechanisms of recanalization, BBBP or the occurrence of HT.

Kim et al (17) performed no-contrast computerized tomography (CT) scans following arterial thrombolysis in patients with acute cerebral infarction and attempted to assess the risk of HT by analyzing the contrast agent retention. This method exhibited good sensitivity but relatively low specificity in predicting the occurrence of HT (18). Previously, related studies have been completed and these have revealed that while a patient may exhibit retention of contrast at multiple sites, the outcomes at each site may be different, suggesting that this method may not be suitable for application to the clinical setting (19). Furthermore, this technique is utilized following arterial thrombolysis, which precludes its application in therapy decision and in patients undergoing intravenous thrombolysis. However, it has been indicated that the aforementioned phenomena is associated with the disruption of the $\mathrm{BBB}$ and HT (19). A previous study demonstrated that HT induced by ischemic stroke starts from the microvasculature (20), and the BBB defect is a key step during HT. In addition, an increasing number of reports confirm that the administration of tPA is one of the risk factors for HT, and that tPA itself and the accompanying reperfusion injury, is a major aggravating factor for the development of the BBB defect $(21,22)$.

Enhanced MR may predict HT effectively $(23,24)$, and HT is associated with the early endothelial damage-induced increase in BBBP and the passage of small molecules (such as gadopentetic acid-DTPA contrast agent or various small proteins) through the $\mathrm{BBB}$ and into the brain tissue $(23,24)$. However, BBB damage increases the BBBP and constitutes the theoretical basis for the assessment of HT by permeability imaging (25). The permeability imaging technique is based on the enhanced dynamic sequences, which allows for the long-term observation of the process of contrast entering in and out of blood vessels through BBB, after the contrast agents have been injected into the blood and thus, provide an improved quantitative assessment (24).

Thus, permeability imaging may help physicians to make clinical decisions. For example, patients with low risk in HT may receive thrombolysis treatment through the time window that has been extended by $3-4.5 \mathrm{~h}$ (26). Certain patients cannot be given thrombolysis treatment even within the time window due to the high risk in HT.

The lesions of acute cerebral infarction, which are different from the brain tumors or the inflammation-related diseases, have insufficient blood supply or may even have no effective blood flow. There may be little or no contrast agent retention in severely ischemic areas due to the lack of blood supply, which may result in failure of detecting the BBB disruption; therefore, the permeability imaging methods (such as dynamic contrast enhanced MR) cannot accurately identify the BBB defect in severely hypoperfused regions (specifically, infarct core areas). However, such areas are the exact HT predilection sites following recanalization. Thus, a mistaken conclusion may be deduced and HT may occur in brain areas without $\mathrm{BBB}$ defect, and reperfusion damage-induced $\mathrm{BBB}$ defect is the only initiator of HT.

Areas with increased BBBP, as detected by permeability imaging, are the exact areas with blood perfusion or relatively adequate collateral circulation. If the ischemia or reperfusion-induced damage is not severe enough, the risk of HT may not increase even with a BBB defect and an elevated BBBP, and patient prognosis may be improved.

The BBBP detected by permeability imaging may fluctuate with the perfusion level and does not always reflect the true extent of BBB damage. Thus, the increase in BBBP values detected by permeability imaging cannot be depended upon for assessment of HT risk in patients with acute cerebral infarction, and further study is required. The permeability imaging and perfusion imaging should be combined to adjust the degree of BBB damage.

\section{Assessment of intracranial collateral circulation}

When acute or chronic severe vascular stenosis and occlusion occur, collateral vessels offer alternative routes for the maintenance of cerebral circulation. Patients with acute cerebral infarction with good compensatory collateral circulation exhibit smaller areas of low perfusion, longer penumbral survival and marked improvement in early clinical symptoms (27). The adequacy of the collateral circulation may help predict the efficacy of intravascular treatments, the final infarct volume and the risk of HT $(28,29)$.

There are various methods for assessing the intracranial collaterals, including the transcranial Doppler (TCD) ultrasound (30), the digital subtraction angiography (DSA) (31), MR angiography (MRA) and CT angiography (CTA) (32). In the assessment of the collateral circulation in patients with carotid artery stenosis or occlusion, TCD has a low cost-performance ratio and thus, may be used for population screening or preliminary diagnosis in local hospitals. However, the results of TCD 
are susceptible to subjective influences and vary with the operator and are therefore not highly repeatable, because the blood flow is small in the collateral vessel and it is difficult to tell the value exactly. In addition, TCD is only able to evaluate Level I collaterals (30). DSA is the gold standard for evaluating Level II and Level III intracranial collaterals. The high spatial resolution of DSA enables clear imaging of the small branches of the vascular network and intra- and extra-cranial collateral vessels, in addition to dynamic observations of the intracranial blood circulation. However, since the DSA is an invasive examination, there is risk of radiation exposure and iodine allergy. In addition, the DSA surgery may induce vasospasm and ischemic stroke, making it inappropriate for dynamic observation in infants, young children, high-risk patients and patients with complex intracranial arterial stenosis (such as Moyamoya disease) (33).

The conventional MRA may accurately evaluate the primary collateral circulation-the circle of Willis (32). It has been reported that the MRA detection rate for the anterior communicating artery is $89.2 \%$ and that for the posterior communicating artery is $81.3 \%$ (21). In MRA Source Imaging (MRA-SI), the minimum vessel diameter that may be displayed is $1 \mathrm{~mm}$ and the specificity of the maximum intensity projection (MIP) is even higher, compared with MRA-SI (34). However, due to the limitation of the anatomical resolution, conventional MRA may only be used for vessels proximal to the circle of Willis; it cannot accurately evaluate the secondary collaterals or the tertiary collaterals (35). Following large intracranial artery occlusion, fluid-attenuated inversion recovery images indicate high-intensity signals in vessels in the brain sulci, which represent the reverse blood flow following the opening of the leptomeningeal collaterals, which is known as the 'ivy sign', an indirect sign of the distal collateral blood flow (36). However, this sign is not reliable and its qualitative and quantitative values are not significant (36). The development of perfusion MR makes the quantitative evaluation of intracranial distal collateral circulations possible. Wu et al (37) used Vessel Encoding Arterial Spin Labeling (VE-ASL) to evaluate patients with internal carotid artery stenosis or occlusion and revealed that it may adequately display leptomeningeal collaterals. Chang et al (38) also provided a similar conclusion, using a territorial ASL technique. However, preliminary findings indicate more unsatisfactory results with ASL, compared with DSA, various results being misleading during the assessment of complex arterial lesions (such as in Moyamoya disease) (39). The study by Chen et al (40) also indicated the absence of higher accuracy during evaluation using ASL in patients with multiple intracranial arterial stenosis or occlusion compared with single artery lesions.

CTA has higher spatial resolution than MRA and is more suited for the evaluation of the circle of Willis (39). The MIP of CTA may clearly display leptomeningeal collaterals and CTA source images provide information regarding certain secondary and tertiary collateral circulations. A systematic retrospective analysis (40) suggested that the information on collateral circulation obtained by CT scanning is most consistent with those taken by DSA, and the difference between observers was also relatively small, therefore a number of early international studies have used these two methods $(40,41)$. Dynamic CTA (or 4D-CTA) is a novel technology that has been utilized in the dynamic evaluation of cerebral blood flow (42). Using the 320-slice CT scan, it may be possible to obtain information on perfusion in addition to the state of the entire intracerebral circulation at the same time, and the imaging quality is almost comparable with the quality of DSA, which makes the clinical application of 4D-CTA promising (42). Currently, a number of international clinical studies (such as the INSPIRE study) are using this technique to investigate the collateral circulation and cerebral perfusion, in addition to other aspects such as intracranial aneurysm and cerebral arteriovenous malformation $(33,41)$. The 4D-CTA has a number of disadvantages, including a large one-time radiation dose of the contrast agent. With the large amount of information acquired, the data processing is also time consuming. Additionally, the results are not intuitive due to the tortuous vessels. There are large differences between operators, and there is difficulty in quantifying the evaluation on the collateral circulation. Its significance in the evaluation of tertiary collaterals is not clear.

In a preliminary study using 4D-CTA, the authors of the present study identified that, in patients with chronic intracranial arterial stenosis or occlusion, there was significant retention of the contrast agent in the brain and the peak time was significantly postponed (40). This is potentially because of a slow reverse of blood flow due to the increased number of newborn vessels and an imperfect $\mathrm{BBB}$, which cause passage of the contrast agent across the $\mathrm{BBB}$ and into the brain tissue. This phenomenon poses the question whether the permeability imaging techniques that can detect changes in the BBBP may be used for evaluating the intracranial secondary and tertiary collateral circulations. Pathological studies suggested that in Moyamoya disease, the formation of meningeal collateral circulation should be associated with immature newborn vessels, which formed anastomoses across the dura and thus resulted in an imperfect BBB $(43,44)$. This finding supports the potential for using permeability imaging to directly assess collateral circulations.

Chen et al (27) has demonstrated good consistency between permeability imaging and DSA in evaluating collateral circulations patients following ischemic stroke. It has demonstrated that the newborn vessels comprise the intracranial tertiary collaterals. Angiogenesis is a complex and dynamic process. Therefore, new capillaries were either born from existing blood vessels or are formed de novo (45). The BBB of newly formed vessels was imperfect, had higher permeability and took weeks to become functionally intact (45), all of which may be evaluated by dynamic contrast-enhanced imaging (46). A previous study used MR to detect angiogenesis by monitoring blood volume changes, which, over time, may reflect the growth of newly formed vessels (46). The measurement of blood volume by perfusion MR was correlated with the density of microvessels, detected by histochemistry. Increased cerebral blood volume (CBV) was correlated with the vascular density in glioma patients (47); however, there is an inherent problem in the association between $\mathrm{CBV}$ and revascularization. Since the increase in the diameter of blood vessels may also lead to an increased blood volume, CBV cannot fully reflect the increase in the number of vessels, as determined by the increase in vascular density on imaging (47).

While studying vascular endothelial growth factor (VEGF), Dvorak et al (48) established an association between angiogenesis and vascular permeability. Once the VEGF receptor is 
activated by VEGF, the permeability of the microvasculature to plasma proteins and other macromolecules is increased. Newborn vessels in tumors, healing wounds, retinopathy, inflammation and the ovaries during ovulation, all exhibit relatively high permeability (49). Quantitative assessment of the quantity of contrast agent passing through the BBB has been used to measure vascular permeability in patients with cervical cancer (50) and glioma tumors (51). CBV, cerebral blood flow $(\mathrm{CBF})$, and volume transfer constant $\left(\mathrm{K}^{\text {trans }}\right)$ in MR have all been used to measure post-stroke angiogenesis (52). In a rat stroke model, the combination of MR and 3D laser-scanning confocal microscopy (LSCM) revealed enhanced signals of newborn vessels at the boundary of the ischemic regions following cell-based therapy and neural progenitor cell treatment, which was confirmed by the increased vascular density and the detection of large thin-walled mother vessels in LSCM (53). The increased angiogenesis was consistent with the increases in CBF and CBV that occur at 6 weeks following therapy and the transient increase in the $\mathrm{K}^{\mathrm{trans}}$, which peaked 1-3 weeks after the cell therapy (54). Compared with animals treated with nerve reconstruction therapy, untreated animals exhibited a delay in the peak of the BBB permeability for 2-5 weeks (55). Previous studies have also demonstrated that angiogenesis in the penumbra started from $12 \mathrm{~h}$ after occlusion and lasted $>21$ days $(56,57)$. The $\mathrm{K}^{\text {trans }}$ map of the tumors, which sustained increase in the signals, represented the continuing growth of new blood vessels (51). By contrast, animals with stroke only exhibited a transient rise of the $\mathrm{K}^{\text {trans }}$ following nerve reconstruction treatment (53). Thus, during post-ischemic recovery, the sensitivity of $\mathrm{K}^{\text {trans }}$ for the detection of angiogenesis is time-dependent.

In addition to $\mathrm{K}^{\text {trans }}$, susceptibility weighted imaging (SWI) is also highly sensitive to the detection of angiogenesis. Since angiogenesis primarily occurs in areas with high oxygen uptake, this may occur in the penumbra and SWI is able to produce early images of small draining veins in the penumbra. Sequence T2 start $\left(\mathrm{T}^{*}\right)$ can detect deoxygenated hemoglobin. Thus, combining SWI, T2* and $\mathrm{K}^{\text {trans }}$ may provide phase information of angiogenesis (55). Physicians are typically unfamiliar with the methods of $\mathrm{K}^{\text {trans }}$ measurements and as a result, use perfusion imaging rather than permeability imaging. However, permeability imaging contains both permeability and perfusion information, which may objectively evaluate the collateral circulation.

\section{Conclusions}

In conclusion, the present review has suggested that the distribution of intensity for previously introduced permeability maps may be used to predict future hemorrhagic transformation in acute ischemic stroke patients treated with tPA or recanalization therapy. The generalization of such a predictive model to produce continuous risk maps would bring additional information during the clinical decision-making process. Specifically, it would help identify patients without BBB abnormalities, which may be a criterion to extend the time window to administer tPA following acute ischemic stroke, in patients who would currently be excluded by the fixed time window. Additionally, the permeability imaging may also be used to evaluate collateral circulation in the perfusion and in the permeability of the ischemic area of the brain. In the future, the permeability imaging may have an improved application to ischemic stroke.

\section{Acknowledgements}

The present review was supported by the National Natural Science Foundations of China (grant nos. 81371286 and 81501024) and Beijing Natural Science Foundation (grant no. 7163226$)$.

\section{References}

1. Milej D, Abdalmalak A, Desjardins L, Ahmed H, Lee TY, Diop M and Lawrence KS: Quantification of blood-brain barrier permeability by dynamic contrast-enhanced NIRS. Sci Rep 7: 1702, 2017.

2. Villringer K, Sanz Cuesta BE, Ostwaldt AC, Grittner U, Brunecker P, Khalil AA, Schindler K, Eisenblätter O, Audebert H and Fiebach JB: DCE-MRI blood-brain barrier assessment in acute ischemic stroke. Neurology 88: 433-440, 2017.

3. Yang GY and Betz AL: Reperfusion-induced injury to the blood-brain barrier after middle cerebral artery occlusion in rats. Stroke 25: 1658-1665, 1994.

4. Hoffmann A, Zhu G and Wintermark M: Advanced neuroimaging in stroke patients: Prediction of tissue fate and hemorrhagic transformation. Expert Rev Cardiovasc Ther 10: 515-524, 2012.

5. Hatashita S and Hoff JT: Brain edema and cerebrovascular permeability during cerebral ischemia in rats. Stroke 21: 582-588, 1990.

6. Sun FL, Wang W, Cheng H, Wang Y, Li L, Xue JL, Wang XF, Ai HX, Zhang L, Xu JD, et al: Morroniside improves microvascular functional integrity of the neurovascular unit after cerebral ischemia. PLoS One 9: e101194, 2014.

7. Liebeskind DS, Kim D, Starkman S, Changizi K, Ohanian AG, Jahan R and Viñuela F: Collateral failure? Late mechanical thrombectomy after failed intravenous thrombolysis. J Neuroimaging 20: 78-82, 2010.

8. Hacke W, Furlan AJ, Al-Rawi Y, Davalos A, Fiebach JB, Gruber F, Kaste M, Lipka LJ, Pedraza S, Ringleb PA, et al: Intravenous desmoteplase in patients with acute ischaemic stroke selected by MRI perfusion-diffusion weighted imaging or perfusion CT (DIAS-2): A prospective, randomised, double-blind, placebo-controlled study. Lancet Neurol 8: 141-150, 2009.

9. Shobha N, Buchan AM and Hill MD; Canadian Alteplase for Stroke Effectiveness Study (CASES): Thrombolysis at 3-4.5 hours after acute ischemic stroke onset-evidence from the Canadian Alteplase for Stroke Effectiveness Study (CASES) registry. Cerebrovasc Dis 31: 223-228, 2011.

10. Smith WS, Sung G, Starkman S, Saver JL, Kidwell CS, Gobin YP, Lutsep HL, Nesbit GM, Grobelny T, Rymer MM, et al: Safety and efficacy of mechanical embolectomy in acute ischemic stroke: Results of the MERCI trial. Stroke 36: 1432-1438, 2005.

11. Smith WS, Sung G, Saver J, Budzik R,Duckwiler G, Liebeskind DS, Lutsep HL, Rymer MM, Higashida RT, Starkman S, et al: Mechanical thrombectomy for acute ischemic stroke: Final results of the Multi MERCI trial. Stroke 39: 1205-1212, 2008.

12. Zhang J, Yang Y, Sun H and Xing Y: Hemorrhagic transformation after cerebral infarction: Current concepts and challenges. Ann Transl Med 2: 81, 2014.

13. Hafez S, Hoda MN, Guo X, Johnson MH, Fagan SC and Ergul A: Comparative analysis of different methods of Ischemia/Reperfusion in hyperglycemic stroke outcomes: Interaction with tPA. Transl Stroke Res 6: 171-180, 2015.

14. Souza LC, Payabvash S, Wang Y, Kamalian S, Schaefer P, Gonzalez RG, Furie KL and Lev MH: Admission CT perfusion is an independent predictor of hemorrhagic transformation in acute stroke with similar accuracy to DWI. Cerebrovasc Dis 33: $8-15,2012$.

15. Furtado AD, Adraktas DD, Brasic N, Cheng SC, Ordovas K, Smith WS, Lewin MR, Chun K, Chien JD, Schaeffer S and Wintermark M: The triple rule-out for acute ischemic stroke: Imaging the brain, carotid arteries, aorta, and heart. AJNR Am J Neuroradiol 31: 1290-1296, 2010.

16. Kaesmacher J, Kaesmacher M, Maegerlein C, Zimmer C, Gersing AS, Wunderlich S, Friedrich B, Boeckh-Behrens T and Kleine JF: Hemorrhagic transformations after thrombectomy: Risk factors and clinical relevance. Cerebrovasc Dis 43: 294-304, 2017. 
17. Kim JM, Park KY, Lee WJ, Byun JS, Kim JK, Park MS, Ahn SW and Shin HW: The cortical contrast accumulation from brain computed tomography after endovascular treatment predicts symptomatic hemorrhage. Eur J Neurol 22: 1453-1458, 2015.

18. Zhu G, Michel P, Zhang W and Wintermark M: Standardization of stroke perfusion CT for reperfusion therapy. Transl Stroke Res 3: 221-227, 2012.

19. Durst C, Monteith S, Sheehan J, Moldovan K, Snell J, Eames M, Huerta T, Walker W, Viola F, Kassell N and Wintermark M: Optimal imaging of in vitro clot sonothrombolysis by MR-guided focused ultrasound. J Neuroimaging 23: 187-191, 2013.

20. Hamann GF, Okada Y and del Zoppo GJ: Hemorrhagic transformation and microvascular integrity during focal cerebral ischemia/reperfusion. J Cereb Blood Flow Metab 16: 1373-1378, 1996.

21. Dijkhuizen RM, Asahi M, Wu O, Rosen BR and Lo EH Rapid breakdown of microvascular barriers and subsequent hemorrhagic transformation after delayed recombinant tissue plasminogen activator treatment in a rat embolic stroke model. Stroke 33: 2100-2104, 2002.

22. Kelly MA, Shuaib A and Todd KG: Matrix metalloproteinase activation and blood-brain barrier breakdown following thrombolysis. Exp Neurol 200: 38-49, 2006

23. Jiang Q, Zhang RL, Zhang ZG, Knight RA, Ewing JR, Ding G, Lu M, Arniego P, Zhang L, Hu J, et al: Magnetic resonance imaging characterization of hemorrhagic transformation of embolic stroke in the rat. J Cereb Blood Flow Metab 22: 559-568, 2002.

24. Jiang Q, Ewing JR, Ding GL, Zhang L, Zhang ZG, Li L, Whitton P, Lu M, Hu J, Li QJ, et al: Quantitative evaluation of BBB permeability after embolic stroke in rat using MRI. J Cereb Blood Flow Metab 25: 583-592, 2005

25. Amtul Z and Hepburn JD: Protein markers of cerebrovascular disruption of neurovascular unit: Immunohistochemical and imaging approaches. Rev Neurosci 25: 481-507, 2014

26. Hacke W, Kaste M, Bluhmki E, Brozman M, Dávalos A, Guidetti D, Larrue V, Lees KR, Medeghri Z, Machnig T, et al: Thrombolysis with Alteplase 3 to 4.5 hours after acute ischemic stroke. N Engl J Med 359: 1317-1329, 2008

27. Chen H, Wu B, Liu N, Wintermark M, Su Z, Li Y, Hu J, Zhang Y, Zhang $\mathrm{W}$ and Zhu G: Using standard first-pass perfusion computed tomographic data to evaluate collateral flow in acute ischemic stroke. Stroke 46: 961-967, 2015.

28. Zaidat OO, Lazzaro MA, Liebeskind DS, Janjua N, Wechsler L, Nogueira RG, Edgell RC, Kalia JS, Badruddin A, English J, et al: Revascularization grading in endovascular acute ischemic stroke therapy. Neurology 79 (Suppl 1): S110-S116, 2012.

29. Marks MP, Lansberg MG, Mlynash M, Olivot JM, Straka M, Kemp S, McTaggart R, Inoue M, Zaharchuk G, Bammer R, et al: Effect of collateral blood flow on patients undergoing endovascular therapy for acute ischemic stroke. Stroke 45: 1035-1039, 2014.

30. Guan J, Zhang S, Zhou Q, Li C and Lu Z: Usefulness of transcranial Doppler ultrasound in evaluating cervical-cranial collateral circulations. Interv Neurol 2: 8-18, 2013.

31. Kortman HG, Smit EJ, Oei MT, Manniesing R, Prokop M and Meijer FJ: 4D-CTA in neurovascular disease: A review. AJNR Am J Neuroradiol 36: 1026-1033, 2015.

32. Stolz E, Mendes I, Gerriets T and Kaps M: Assessment of intracranial collateral flow by transcranial color-coded duplex sonography using a temporal and frontal axial insonation plane. J Neuroimaging 12: 136-143, 2002.

33. Roach BA, Donahue MJ, Davis LT, Faraco CC, Arteaga D, Chen SC, Ladner TR, Scott AO and Strother MK: Interrogating the functional correlates of collateralization in patients with intracranial stenosis using multimodal hemodynamic imaging. AJNR Am J Neuroradiol 37: 1132-1138, 2016.

34. Seidel G, Kaps M and Gerriets T: Potential and limitations of transcranial color-coded sonography in stroke patients Stroke 26: 2061-2066, 1995.

35. Hernández-Pérez M, Puig J, Blasco G, Pérez de la Ossa N, Dorado L, Dávalos A and Munuera J: Dynamic magnetic resonance angiography provides collateral circulation and hemodynamic information in acute ischemic stroke. Stroke 47: 531-534, 2016.

36. Kaku Y, Iihara K, Nakajima N, Kataoka H, Fukushima K, Iida $H$ and Hashimoto N: The leptomeningeal ivy sign on fluid-attenuated inversion recovery images in moyamoya disease: Positron emission tomography study. Cerebrovasc Dis 36: 19-25, 2013.
37. Wu B, Wang X, Guo J, Xie S, Wong EC, Zhang J, Jiang X and Fang J: Collateral circulation imaging: MR perfusion territory arterial spin-labeling at 3T. AJNR Am J Neuroradiol 29: $1855-1860,2008$

38. Chang SM, Petersen ET, Zimine I, Sitoh YY, Lim CC and Golay X: Territorial arterial spin labeling in the assessment of collateral circulation: Comparison with digital subtraction angiography. Stroke 39: 3248-3254, 2008.

39. McVerry F, Liebeskind DS and Muir KW: Systematic review of methods for assessing leptomeningeal collateral flow. AJNR Am J Neuroradiol 33: 576-582, 2012.

40. Chen H, Wu B, Zhu G, Wintermark M, Wu X, Su Z, Xu X, Tian C, Ma L, Zhang W and Lou X: Permeability imaging as a biomarker of leptomeningeal collateral flow in patients with intracranial arterial stenosis. Cell Biochem Biophys 71: 1273-1279, 2015.

41. Ono Y, Abe K, Suzuki K, Iimura H, Sakai S, Uchiyama S and Okada Y: Usefulness of 4D-CTA in the detection of cerebral dural sinus occlusion or stenosis with collateral pathways. Neuroradiol J 26: 428-438, 2013.

42. Sheth SA and Liebeskind DS: 'Imaging evaluation of collaterals in the brain: Physiology and clinical translation'. Curr Radiol Rep 2: 29, 2014.

43. Lim M, Cheshier S and Steinberg GK: New vessel formation in the central nervous system during tumor growth, vascular malformations, and Moyamoya. Curr Neurovasc Res 3: 237-245, 2006.

44. Ding G, Jiang Q, Li L, Zhang L, Gang Zhang Z, Ledbetter KA, Ewing JR, Li Q and Chopp M: Detection of BBB disruption and hemorrhage by Gd-DTPA enhanced MR after embolic stroke in rat. Brain Res 1114: 195-203, 2006.

45. Vallon M, Chang J, Zhang H and Kuo CJ: Developmental and pathological angiogenesis in the central nervous system. Cell Mol Life Sci 71: 3489-3506, 2014.

46. Moisan A, Favre IM, Rome C, Grillon E, Naegele B, Barbieux M, De Fraipont F, Richard MJ, Barbier EL, Remy C and Detante O: Microvascular plasticity after experimental stroke: A molecular and MRI study. Cerebrovasc Dis 38: 344-353, 2014.

47. Darpolor MM, Molthen RC and Schmainda KM: Multimodality imaging of abnormal vascular perfusion and morphology in preclinical 9L gliosarcoma model. PLoS One 6: e16621, 2011.

48. Dvorak HF, Nagy JA, Feng D, Brown LF and Dvorak AM: Vascular permeability factor/vascular endothelial growth factor and the significance of microvascular hyperpermeability in angiogenesis. Curr Top Microbiol Immunol 237: 97-132, 1999.

49. Neeman M: Functional and molecular MR imaging of angiogenesis: Seeing the target, seeing it work. J Cell Biochem Suppl 39: 11-17, 2002.

50. Dickie BR, Rose CJ, Kershaw LE, Withey SB, Carrington BM, Davidson SE, Hutchison G and West CML: The prognostic value of dynamic contrast-enhanced MRI contrast agent transfer constant $\mathrm{K}^{\text {trans }}$ in cervical cancer is explained by plasma flow rather than vessel permeability. Br J Cancer 116: 1436-1443, 2017.

51. Jain R: Measurements of tumor vascular leakiness using DCE in brain tumors: Clinical applications. NMR Biomed 26: 1042-1049, 2013.

52. Seevinck PR, Deddens LH and Dijkhuizen RM: Magnetic resonance imaging of brain angiogenesis after stroke. Angiogenesis 13: 101-111, 2010.

53. Jiang Q, Zhang ZG, Ding GL, Zhang L, Ewing JR, Wang L, Zhang $\mathrm{R}, \mathrm{Li} \mathrm{L}, \mathrm{Lu} \mathrm{M}$, Meng H, et al: Investigation of neural progenitor cell induced angiogenesis after embolic stroke in rat using MRI. Neuroimage 28: 698-707, 2005.

54. Zhang ZG, Zhang L, Jiang Q and Chopp M: Bone marrow-derived endothelial progenitor cells participate in cerebral neovascularization after focal cerebral ischemia in the adult mouse. Circ Res 90: 284-288, 2002.

55. Ding G, Jiang Q, Li L, Zhang L, Zhang ZG, Ledbetter KA, Gollapalli L, Panda S, Li Q, Ewing JR and Chopp M: Angiogenesis detected after embolic stroke in rat brain using magnetic resonance T2*WI. Stroke 39: 1563-1568, 2008.

56. Yano A, Shingo T, Takeuchi A, Yasuhara T, Kobayashi K, Takahashi K, Muraoka K, Matsui T, Miyoshi Y, Hamada H and Date I: Encapsulated vascular endothelial growth factor-secreting cell grafts have neuroprotective and angiogenic effects on focal cerebral ischemia. J Neurosurg 103: 104-114, 2005.

57. Slevin M, Kumar P, Gaffney J, Kumar S and Krupinski J: Can angiogenesis be exploited to improve stroke outcome? Mechanisms and therapeutic potential. Clin Sci (Lond) 111: 171-183, 2006. 